VOL. $19(1978), 29-37$.

\title{
On the rate of convergence of interpolation polynomials of Hermite-F ejér type
}

\section{J. Prasad}

For the interpolation polynomial of Hermite-Fejêr type $A_{n}[f]$ of degree less than or equal to $4 n-1$ constructed on the nodes. $x_{k}=\cos \frac{2 k-1}{2 n} \pi, k=1,2, \ldots, n$, it is shown that for $f \in C_{M}(\Omega)$ the inequality

$$
\left|A_{n}[f](x)-f(x)\right| \leq \frac{c_{3}^{M}}{n} \sum_{k=1}^{n} \Omega\left(\frac{\sqrt{1-x^{2}}}{k}+\frac{1}{k^{2}}\right), \quad-1 \leq x \leq 1,
$$

holds where $C_{M}(\Omega)$ is the class of continuous functions on $[-1,1]$ satisfying certain conditions, $\Omega$ is a certain modulus of continuity, and $c_{3}$ and $M$ are positive constants.

The Hermite-Fejér interpolation polynomial $H_{n}[f]$ of degree less than or equal to $2 n-1$ of a function $f$ defined on $[-1,1]$ is given by

$$
H_{n}[f](x)=\sum_{k=1}^{n} f\left(x_{k n}\right)\left(1-x x_{k n}\right)\left[\frac{T_{n}(x)}{n\left(x-x_{k n}\right)}\right]^{2}
$$

where

$$
x_{k n}=\cos \frac{2 k-1}{2 n} \pi, k=1,2, \ldots, n,
$$

are the zeros of the Chebyshev polynomial ${ }_{n}(x)=\cos (n \arccos x)$. Fejér Received 16 May 1978. 
[2] proved that $H_{n}[f]$ converges uniformly to $f$ on $[-1,1]$ provided $f$ is continuous on $[-1,1]$. For the rate of convergence Moldovan [6] obtained the estimate

$$
\left\|H_{n}[f]-f\right\| \leq 2 \pi \omega_{f}\left(\frac{\log n}{n}\right), n \geq 4,
$$

where $\|f\|=\max _{-1 \leq x \leq 1}|f(x)|$ and $\omega_{f}$ is the modulus of continuity of $f(x)$. This estimate was also established by Shisha and Mond [8].

Let $\Omega$ be an increasing, subadditive, and continuous function on $\{x: x \geq 0\}$ with $\Omega(0)=0$, and let $C_{M}(\Omega)$ be the class of continuous functions on $[-1,1]$ defined by

$$
f \in C_{M}(\Omega) \Leftrightarrow \omega_{f}(h) \leq M \Omega(h)
$$

or equivalently, $f \in C_{M}(\Omega)$ iff $\left|f\left(x^{\prime}\right)-f\left(x^{\prime \prime}\right)\right| \leq M \Omega\left(\left|x^{\prime}-x^{\prime \prime}\right|\right)$ for all $x^{\prime}, x^{\prime \prime} \in[-1,1]$. We have then the following result due to Bojanic [1].

THEOREM 1 (Bojanic). There exist constants $c$ and $C$ $(0<c<C<\infty)$ such that for $n \geq 2$,

$$
\frac{c M}{n} \sum_{k=2}^{n} \Omega\left(\frac{1}{k}\right) \leq \sup _{f \in C_{M}(\Omega)}\left\|H_{n}[f]-f\right\| \leq \frac{C M}{n} \sum_{k=1}^{n} \Omega\left(\frac{1}{k}\right) .
$$

Saxena [7] showed that a better local approximation can be obtained at the end points of the interval by proving the following:

THEOREM 2. There exists a constant $C^{*}$ such that for $n \geq 2$ and $-1 \leq x \leq 1$,

$$
\left|H_{n}[f](x)-f(x)\right| \leq \frac{C^{*} M}{n} \sum_{k=1}^{n} \Omega\left(\frac{\left(1-x^{2}\right)^{\frac{1}{2}}}{k}+\frac{1}{k^{2}}\right) .
$$

Recently Stancu [9] has considered the polynomial $A_{n}[f]$ of degree less than or equal to $4 n-1$ uniquely determined by the conditions:

$$
\begin{aligned}
A_{n}[f]\left(x_{i n}\right) & =f\left(x_{i n}\right), k=1,2, \ldots, n ; \\
A_{n}^{(i)}[f]\left(x_{k n}\right) & =0, i=1,2,3, k=1,2, \ldots, n .
\end{aligned}
$$


Stancu showed that $A_{n}[f]$ converges uniformly to $f$ on $[-1,1]$ if $f$ is continuous on $[-1,1]$. He also proved that

$$
\left\|A_{n}[f]-f\right\|=O(1) \omega_{f}\left(n^{-\frac{1}{2}}\right),
$$

where the $O(1)$ is independent of $f$ and $n$. Later Florica [3] improved the above result ( 7 ), showing that

$$
\left\|A_{n}[f]-f\right\|=O(1) \omega_{f}\left(\frac{\log n}{n}\right) \text {, for } n>1 \text {. }
$$

This result of Florica was further improved by Mills [5] who proved the following:

THEOREM 3 (Mills). There are positive constants $c_{1}$ and $c_{2}$ such that for $n>1$,

$$
\frac{c_{1}}{n} \sum_{r=2}^{n} \Omega\left(\frac{1}{r}\right) \leq \sup _{f \in C(\Omega)}\left\|A_{n}[f]-f\right\| \leq \frac{c_{2}}{n} \sum_{r=1}^{n} \Omega\left(\frac{1}{r}\right) .
$$

Our aim here is to show that a better local approxination can be obtained in this case also. For this purpose we shall prove the following:

THEOREM 4. There exists a constant $c_{3}$ such that for $n \geq 2$ and $-1 \leq x \leq 1$,

$$
\left|A_{n}[f](x)-f(x)\right| \leq \frac{c_{3}^{M}}{n} \sum_{k=1}^{n} \Omega\left(\frac{\left(1-x^{2}\right)^{\frac{1}{2}}}{k}+\frac{1}{k^{2}}\right) .
$$

Stancu [9] has proved that

$$
A_{n}[f](x)=\sum_{k=1}^{n} f\left(x_{k}\right) \rho_{k}(x),
$$

where

$$
\begin{aligned}
& \rho_{k}(x)=u_{k}(x)+v_{k}(x)+w_{k}(x), \\
& u_{k}(x)=n^{-4}\left(1-x_{k}^{2}\right)\left(1-x^{2}\right)\left[\frac{T_{n}(x)}{x-x_{k}}\right]^{4}, \\
& v_{k}(x)=\frac{n^{-4}}{6}\left(4 n^{2}-1\right)\left(x-x_{k}\right)^{2}\left(1-x x_{k}\right)\left[\frac{n_{n}(x)}{x-x_{k}}\right]^{4},
\end{aligned}
$$




$$
w_{k}(x)=\frac{n^{-4}}{2}\left[\frac{T^{2}(x)}{x-x_{k}}\right]^{2},
$$

and $x_{k}=x_{k n}$ are as in (2). Now we shall prove the following:

LEMMA. If

$$
\frac{j-1}{n} \pi \leq \theta \leq \frac{j}{n} \pi \quad(j=1,2, \ldots, n), x=\cos \theta,
$$

then

$$
\rho_{k}(x) \leq \begin{cases}19, & \text { if } k=j, \\ \frac{70}{\left(i-\frac{2}{2}\right)^{2}}, & \text { if } j<k=j+i \leq n \text { or } 1 \leq k=j-i<j .\end{cases}
$$

Proof. Putting $x=\cos \theta, 0 \leq \theta \leq \pi, x_{k}=\cos \theta_{k}, \theta_{k}=\frac{2 k-1}{2 n} \pi$, $k=1,2, \ldots, n$, and using the definition of $j$ we see that

$$
\frac{1}{\sin \frac{1}{2}\left|\theta_{k}-\theta\right|} \leq \frac{n}{\left(i-\frac{1}{2}\right)} \text {, for } k \neq j
$$

Further from (11), (12), (13), (14), and (15) it follows that

$$
\begin{aligned}
\rho_{k}(x)= & n^{-4}\left(1-\cos ^{2} \theta_{k}\right)\left(1-\cos ^{2} \theta\right)\left[\frac{\cos n \theta}{\cos \theta-\cos \theta_{k}}\right]^{4} \\
& +\frac{n^{-4}}{6}\left(4 n^{2}-1\right)\left(\cos \theta-\cos \theta_{k}\right)^{2}\left(1-\cos \theta \cos \theta_{k}\right)\left[\frac{\cos n \theta}{\cos \theta-\cos \theta_{k}}\right]^{4} \\
= & +\frac{n^{-4}}{2}\left[\frac{\cos ^{2} n \theta}{\cos \theta-\cos \theta_{k}}\right]^{2}
\end{aligned}
$$

Since

$$
\sin \theta \leq \sin \theta+\sin \theta_{k} \leq 2 \sin \frac{1}{2}\left(\theta+\theta_{k}\right)
$$

and

$$
\sin \theta_{k} \leq \sin \theta_{k}+\sin \theta \leq 2 \sin \frac{2}{2}\left(\theta+\theta_{k}\right) \text {, }
$$


we have after simple computations,

$$
\begin{aligned}
I_{1} & \leq \frac{\left|\cos n \theta-\cos n \theta_{k}\right|^{4}}{n^{4}\left[\sin \frac{1}{2}\left(\theta_{k}-\theta\right)\right]^{4}} \\
& \leq \frac{16}{n^{4}}\left[\frac{\sin \frac{1}{2} n\left(\theta_{k}-\theta\right)}{\sin \frac{7}{2}\left(\theta_{k}-\theta\right)}\right]^{4} .
\end{aligned}
$$

Next we have

$$
\begin{aligned}
I_{2} & \leq \frac{1}{n^{2}}\left(1-\cos \theta \cos \theta_{k}\right)\left[\frac{\cos n \theta}{\cos \theta-\cos \theta_{k}}\right] \\
& \leq \frac{1}{n^{2}}\left(1-\cos \theta \cos \theta_{k}+\sin \theta \sin \theta_{k}\right)\left[\frac{\cos n \theta-\cos n \theta_{k}}{\cos \theta-\cos \theta_{k}}\right]^{2} \\
& \leq \frac{2}{n^{2}}\left[\frac{\sin \frac{3}{2} n\left(\theta_{k}-\theta\right)}{\sin \frac{1}{2}\left(\theta_{k}-\theta\right)}\right]^{2} .
\end{aligned}
$$

Similarly,

$$
\begin{aligned}
I_{3} & \leq \frac{1}{2 n^{4}}\left[\frac{\cos n \theta-\cos n \theta_{k}}{\cos \theta-\cos \theta_{k}}\right]^{2} \\
& =\frac{1}{2 n^{4}}\left[\frac{\sin \frac{1}{2} n\left(\theta+\theta_{k}\right) \sin \frac{1}{2} n\left(\theta_{k}-\theta\right)}{\sin \frac{1}{2}\left(\theta+\theta_{k}\right) \sin \frac{1}{2}\left(\theta_{k}-\theta\right)}\right]^{2} .
\end{aligned}
$$

Consequently from (17), (20), (21), and (22), it follows that

$$
\begin{array}{r}
\rho_{k}(x) \leq \frac{16}{n^{4}}\left[\frac{\sin \frac{1}{2} n\left(\theta_{k}-\theta\right)}{\sin ^{\frac{1}{2}}\left(\theta_{k}-\theta\right)}\right]^{4}+\frac{2}{n^{2}}\left[\frac{\sin \frac{1}{2} n\left(\theta_{k}-\theta\right)}{\sin \frac{1}{2}\left(\theta_{k}-\theta\right)}\right]^{2} \\
\quad+\frac{1}{2 n^{\frac{1}{4}}}\left[\frac{\sin \frac{1}{2}\left(\theta+\theta_{k}\right) \sin \frac{1}{2} n\left(\theta_{k}-\theta\right)}{\sin \frac{1}{2}\left(\theta+\theta_{k}\right) \sin \frac{1}{2}\left(\theta_{k}-\theta\right)}\right]^{2} .
\end{array}
$$

Thus if $k \neq j$, on using the inequality

$$
\frac{1}{\sin \frac{1}{2}\left(\theta+\theta_{k}\right)} \leq \frac{1}{\sin \frac{1}{2}\left|\theta_{k}-\theta\right|}, 0 \leq \theta \leq \pi, 0<\theta_{k}<\pi,
$$

and (16) we obtain, from (23), 
(24)

$$
\begin{aligned}
\rho_{k}(x) & \leq \frac{17}{n^{4}\left[\sin \frac{2}{2}\left(\theta_{k}-\theta\right)\right]^{4}}+\frac{2}{n^{2}\left[\sin \frac{2}{2}\left(\theta_{k}-\theta\right)\right]^{2}} \\
& \leq \frac{70}{\left(i-\frac{3}{2}\right)^{2}} \text {, for } k \neq j .
\end{aligned}
$$

Further if $k=j$ then from (23) we have on using the inequality $|\sin n \theta| \leq n|\sin \theta|$,

$$
\rho_{j}(x) \leq 19
$$

Hence, from (24) and (25), the lemma follows.

Proof of the theorem. For any $f \in C_{M}(\Omega)$ and $x \in[-1,1]$ we have

$$
\begin{aligned}
\left|A_{n}[f](x)-f(x)\right| & \leq \sum_{k=1}^{n}\left|f\left(x_{k}\right)-f(x)\right| \rho_{k}(x) \\
& \leq M \sum_{k=1}^{n} \Omega\left(\left|x-x_{k}\right|\right) \rho_{k}(x) .
\end{aligned}
$$

Putting $x=\cos \theta, 0 \leq \theta \leq \pi, x_{k}=\cos \theta_{k}, \theta_{k}=\frac{2 k-1}{2 n} \pi$, $k=1,2, \ldots, n$, we then have

$$
\left|A_{n}[f](x)-f(x)\right| \leq M \sum_{k=1}^{n} \Omega\left(\left|\cos \theta-\cos \theta_{k}\right|\right) \rho_{k}(x)
$$

Since

$$
\cos \theta-\cos \theta_{k}=\left(\theta_{k}-\theta\right) \sin \theta-\frac{z}{2}\left(\theta_{k}-\theta\right)^{2} \cos \eta, \theta<\eta<\theta_{k},
$$

it follows that

$$
\Omega\left(\left|\cos \theta-\cos \theta_{k}\right|\right) \leq \Omega\left(\left|\theta_{k}-\theta\right| \sin \theta\right)+\Omega\left(\left|\theta_{k}-\theta\right|^{2}\right),
$$

and we obtain

(26) $\quad\left|A_{n}[f](x)-f(x)\right| \leq M \sum_{k=1}^{n}\left[\Omega\left(\left|\theta_{k}-\theta\right| \sin \theta\right)+\Omega\left(\left|\theta_{k}-\theta\right|^{2}\right]\right] \rho_{k}(x)$.

Now if

$$
\frac{j-1}{n} \pi \leq \theta \leq \frac{j}{n} \pi, j=1,2, \ldots, n,
$$

then from [4] it is known that 
(27)

$$
\begin{aligned}
\left|\theta_{k}-\theta\right| & \leq \frac{\pi}{2 n}, \text { if } k=j, \\
& \leq \frac{3 i \pi}{2 n}, \text { if } j<k=j+i \leq n \text { or } 1 \leq k=j-i<j .
\end{aligned}
$$

From (26), (27), and the lemma it follows that

$$
\begin{aligned}
&\left|A_{n}[f](x)-f(x)\right| \leq 19 M\left[\Omega\left(\frac{\pi \sin \theta}{2 n}\right)\right.\left.+\Omega\left(\frac{\pi^{2}}{4 n^{2}}\right)\right] \\
&+70 M \sum_{k \neq j}\left[\Omega\left(\frac{3 i \pi \sin \theta}{2 n}\right)+\Omega\left(\frac{9 i^{2} \pi^{2}}{4 n^{2}}\right)\right] \frac{1}{\left(i-\frac{1}{2}\right)^{2}} \\
& \leq \lambda M \sum_{r=1}^{n} \frac{1}{r^{2}}\left[\Omega\left(\frac{r+1}{n+1} \pi \sin \theta\right)+\Omega\left(\frac{(r+1)^{2} \pi^{2}}{(n+1)^{2}}\right)\right],
\end{aligned}
$$

where $\lambda$ is a positive constant. On using the inequality (6) of [7] for $m=n+1$, we find that

$$
\sum_{r=1}^{n} \frac{1}{r^{2}} \Omega\left(\frac{r+1}{n+1} \pi \sin \theta\right) \leq \frac{8 \pi}{n+1} \int_{\pi / n+1}^{\pi} \frac{\Omega(t \sin \theta)}{t^{2}} d t
$$

and

$$
\sum_{r=1}^{n} \frac{1}{r^{2}} \Omega\left(\frac{(r+1)^{2} \pi^{2}}{(n+1)^{2}}\right) \leq \frac{8 \pi}{n+1} \int_{\pi / n+1}^{\pi} \frac{\Omega\left(t^{2}\right)}{t^{2}} d t .
$$

Hence (28), (29), and (30) yield

(31) $\left|A_{n}[f](x)-f(x)\right| \leq \frac{8 \pi M \lambda}{n+1}\left[\int_{\pi / n+1}^{\pi} \frac{\Omega(t \sin \theta)}{t^{2}} d t+\int_{\pi / n+1}^{\pi} \frac{\Omega\left(t^{2}\right)}{t^{2}} d t\right]$.

But

$$
\begin{aligned}
\int_{\pi / n+1}^{\pi} \frac{\Omega(t \sin \theta)}{t^{2}} d t & =\frac{1}{\pi} \int_{1}^{n+1} \Omega\left(\frac{\pi \sin \theta}{t}\right) d t \\
& \leq 2 \int_{1}^{2 n} \Omega\left(\frac{\sin \theta}{t}\right) d t \\
& \leq 8 \int_{1}^{n} \Omega\left(\frac{\sin \theta}{t}\right) d t \\
& \leq 8 \sum_{k=1}^{n} \Omega\left(\frac{\sin \theta}{k}\right) .
\end{aligned}
$$




$$
\begin{aligned}
\int_{\pi / n+1}^{\pi} \frac{\Omega\left(t^{2}\right)}{t^{2}} d t & =\frac{1}{\pi} \int_{1}^{n+1} \Omega\left(\frac{\pi^{2}}{t^{2}}\right) d t \\
& \leq \frac{1}{\pi} \int_{1}^{2 n} \Omega\left(\frac{\pi^{2}}{t^{2}}\right) d t \\
& \leq 16 \int_{1}^{n} \Omega\left(\frac{1}{t^{2}}\right) d t \\
& \leq 16 \sum_{k=1}^{n} \Omega\left(\frac{1}{k^{2}}\right) .
\end{aligned}
$$

Consequently from (31), (32), and (33) it follows that

$$
\begin{aligned}
\left|A_{n}[f](x)-f(x)\right| & \leq \frac{256 \pi M \lambda}{n+1}\left[\sum_{k=1}^{n} \Omega\left(\frac{\sqrt{1-x^{2}}}{k}+\frac{1}{k^{2}}\right]\right] \\
& \leq \frac{c_{3}^{M}}{n} \sum_{k=1}^{n} \Omega\left(\frac{\sqrt{1-x^{2}}}{k}+\frac{1}{k^{2}}\right) .
\end{aligned}
$$

This completes the proof of the theorem.

Since the modulus of continuity $\omega_{f}$ of any continuous function $f$ on $[-1,1]$ has the same properties as $\Omega$, we also conclude from the above theorem that for any continuous function $f$ on $[-1,1]$ the estimate

$$
\left|A_{n}[f](x)-f(x)\right| \leq \frac{C_{1}^{*}}{n} \sum_{k=1}^{n} \omega_{f}\left(\frac{\sqrt{1-x^{2}}}{k}+\frac{1}{k^{2}}\right)
$$

is valid for $-1 \leq x \leq 1$.

\section{References}

[1] R. Bojanic, "A note on the precision of interpolation by Hermite-Fejér polynomials", Proc. Conf. Constructive Theory of Functions (Approximation Theory), Budapest, 1969, 69-76 (Akadémiai Kiadó, Budapest, 1972).

[2] Leopold Fejër, "Ueber Interpolation", Nachr. Ges. Wissensch. Göttingen $1916,66-91$. 
[3] Olariu Florica, "Asupra ordinului de aproximatie prin polinoame de interpolare de tip Hermite-Fejér cu noduri cvadruple" [On the order of approximation by interpolating polynomials of HermiteFejér type with quadruple nodes], An. Univ. Timisoara Ser. Sti. Mat.-Fiz. 3(1965), 227-234.

[4] 0. Ниц [0. Kis], "Замечания о порядне сходимости лаграннева ннтерполировання" [Remark on the order of convergence of Lagrange interpolation], Ann. Univ. Sci. Budapest. Eötvös Sect. Math. 11 (1968), 27-40.

[5] T.M. MilIs, "On interpolation polynomials of the Hermite-Fejer type", Colzoq. Math. 35 (1976), 159-163.

[6] Elena Moldovan, "Observatii asupra unor procedee de interpolare generalizate" [Observations on certain generalised interpolation methods], Acad. Repub. Pop. Romîne. Bul. Sti. Sect. Sti. Mat. Fiz. 6 (1954), 477-482.

[7] R.B. Saxena, "A note on the rate of convergence of Hermite-Fejér interpolation polynomials", Canad. Math. Bulz. 17 (1974), 299-301.

[8] 0 . Shisha and $B$. Mond, "The rapidity of convergence of the HermiteFejér approximation to functions of one or several variables", Proc. Amer. Math. Soc. 16 (1965), 1269-1276.

[9] D.D. Stancu, "Asupra unei demonstratii a teoremei lui Weierstrass" [On a proof of the theorem of Weierstrass], Bul. Inst. Politehn. Iasi (N.S.) 5 (9) (1959), 47-50.

Department of Mathematics, California State University, Los Angeles, California, USA. 